\title{
ANÁLISIS DE LA $\beta$-GALACTOSILCERAMIDASA LEUCOCITARIA EN PACIENTES COLOMBIANOS CON SOSPECHA CLÍNICA DE ENFERMEDAD DE KRABBE, UN TAMIZAJE DE ALTO RIESGO
}

\author{
LINA MANUELA JAY GARCIA MSc ${ }^{1}$, \\ ALFREDO URIBE ARDILA MSc. PhD ${ }^{2 *}$, ADIS AYALA FAJARDO MSc ${ }^{3}$ \\ ${ }^{1} M S c .$, Coinvestigadora del Centro de Investigaciones en Bioquímica \\ de la Universidad de los Andes, Bogotá - Colombia \\ ${ }^{2}$ Docente Investigador Universidad de los Andes. \\ Director, Centro de Investigaciones en Bioquímica (CIBI) \\ Departamento de Ciencias Biológicas Universidad de los Andes, Bogotá - Colombia \\ Calle 1 No. 18A - 10, Edificio M - Tercer Piso, Bogotá - Colombia \\ ${ }^{3}$ Docente Investigador de la Universidad Distrital, Bogotá - Colombia
}

*Correspondencia: jeuribe@uniandes.edu.co Recibido: 7 Febrero $2017 \quad$ Aceptado: 3 Abril 2017

\begin{abstract}
RESUMEN
La Enfermedad de Krabbe (EK), es un desorden del metabolismo de esfingolípidos de herencia autosómica recesiva causada por la deficiencia de $\beta$ - galactosilceramidasa ( $\beta$-Galsil) (E.C. 3.2.1.46), defecto enzimático que causa un cuadro neurodegenerativo, hipertonía muscular y espasticidad, convulsiones, pérdida de la audición y en un $85 \%$ de los casos la muerte temprana, entre otros hallazgos.

La incidencia de la EK documentada para Estados Unidos y Europa es de 1:100.000 recién nacidos, pero estudios recientes han demostrado valores mayores de 1:22.000 aproximadamente en New York. En América Latina los informes son escasos, con reportes de tamizaje de alto riesgo en Brasil y casos aislados en México, ofreciendo un panorama de subdiagnóstico importante, situación a la que no es ajena Colombia, donde no hay en la literatura referentes a la enfermedad.

Se presenta entonces a la comunidad científica, un estudio de valores de actividad y de referencia para la enzima $\beta$-Galactosilceramidasa leucocitaria, a partir de 259 muestras de 110 individuos sanos y 149 pacientes con compromiso neurodegenerativo (CND). La valoración enzimática involucró dos métodos (Colorimétrico y Fluorométrico) de punto final que permitieron establecer un rango de referencia para $\beta$-Galsil en técnica Colorimétrica: 2,04 -14,93 $\mathrm{nmol} / \mathrm{mgprot} / \mathrm{h}$ y en técnica fluorométrica: $0,3-4,21 \mathrm{nmol} / \mathrm{mgprot} / \mathrm{h}$. El estudio de tamizaje permitió identificar un paciente afectado con enfermedad de Krabbe quien presentó valores de actividad expresados en nmol/mgprot/h de 1,85 y 0,034 , en forma correspondiente para las técnicas antes descritas. Un hallazgo final que permite validar los dos métodos estandarizados para el diagnóstico de la enfermedad y establecer valores de referencia en población colombiana.
\end{abstract}

Palabras Claves: Enfermedad de Krabbe; Deficiencia de $\beta$ - galactosilceramidasa; Enfermedades lisosomales. 


\title{
ANALYSIS OF $\beta$-GALACTOSILCERAMIDASE LEUKOCYTAL IN COLOMBIAN IN PATIENTS WITH SUSPECT CLINIC OF KRABBE DISEASE, A HIGH - RISK SCREENING
}

\begin{abstract}
Krabbe disease is a disorder of autosomal recessive sphingolipid metabolism caused by deficiency $\beta$-galactosylceramidase ( $\beta$-Galsil) (EC3.2.1.46), an enzymatic defect that causes a hurt neurodegenerative, muscular hypertonia and Spasticity, convulsions, hearing loss and in $85 \%$ of cases early death, among other findings.

The incidence documented for "Krabbe disease" in the United States and Europe is 1:100.000 newborns, but recent studies have shown values greater than 1:22.000 in New York. In Latin America, reports are limited, with reports of high-risk screening in Brazil and isolated cases in Mexico, providing an important underdiagnosis scenario, a situation that is not unknown in Colombia, where there is no literature on the disease.

A study of activity and reference values for $\beta$-galactosylceramidase enzyme leukocytal, was then presented to the scientific community, from 259 samples from 110 healthy people and 149 patients with neurodegenerative compromise. The enzymatic evaluation involved two methods (Colorimetric and Fluorometric) that allowed the establishment of a reference range for $\beta-G a l s i l$ in Colorimetric technique: $2.04-14.93 \mathrm{nmol} / \mathrm{mgprot} / \mathrm{h}$ and in fluorometric technique: $0.3-4.21 \mathrm{nmol} / \mathrm{mgprot} / \mathrm{h}$. The screening study allowed the identification of a patient with Krabbe disease who presented activity values expressed in nmol/mgprot/h of 1.85 and 0.034 , correspondingly to the techniques described above. A final finding that allows to validate the two standardized methods for the diagnosis of the disease and to establish reference values in Colombian population.
\end{abstract}

Keywords: Krabbe disease; deficiency of $\beta$-galactosylceramidase; Lysosomal Disease.

\section{ANÁLISE DE $\beta$-GALACTOSILCERAMIDASE LEUKOCYTAL EM COLOMBIANO EM PACIENTES COM SUSPEITA CLINICA DA DOENÇA DE KRABBE, UMA BLINDAGEM DE ALTO RISCO}

\section{RESUMO}

A doença de Krabbe é uma desordem do metabolismo de esfingolípidos autossômicos recessivos causada pela deficiência $\beta$-galactosilceramidase ( $\beta$-Galsil) (EC 3.2.1.46), um defeito enzimático que causa uma neurodegenerativa relatório, hipertonia muscular e espasticidade, convulsões, perda auditiva e em $85 \%$ Dos casos de morte precoce, entre outros.

A incidência da doença de Krabbe documentado para os Estados Unidos e na Europa é de 1:100.000 recém-nascidos, mas estudos recentes têm mostrado valores maiores do que cerca de 1:22.000, em Nova York. Na América Latina, os relatórios são escassos, com relatos de rastreio de alto risco no Brasil e casos isolados no México, proporcionando um cenário importante subdiagnóstico, a situação não é desconhecida em que a Colômbia, não há literatura Onde sobre a doença.

Um estudo de atividade e valores de referência para a enzima $\beta$-galactosilceramidase leucocital, foi então apresentado à comunidade científica, de 259 amostras de 110 indivíduos saudáveis e 149 pacientes com comprometimento neurodegenerativo. A avaliação enzimática envolveu dois métodos (Colorimétrico e Fluorométrico) que permitiram o estabelecimento de uma gama de referência para $\beta$-Galsil na técnica Colorimétrica: 2,04-14,93 nmol $/ \mathrm{mgprot} / \mathrm{h}$ e na técnica fluorométrica: 0,3-4,21 nmol $/ \mathrm{mgprot} / \mathrm{h}$. O estudo de triagem permitiu a identificação de um paciente com doença de Krabbe que apresentou valores de atividade expressados em nmol / mgprot / h de 1,85 e 0,034, correspondente ao das técnicas descritas acima. Uma conclusão final que valida os dois métodos padronizados para o diagnóstico da doença e estabelecer valores de referência na população colombiano. 


\section{Introducción}

La Enfermedad de Krabbe (EK), es un desorden de herencia autosómica recesiva causada por la deficiencia de D-galactosil-N-acilesfingosina galactohidrolasa (E.C. 3.2.1.46) (1,2), conocida comúnmente como $\beta$ - galactosilceramidasa ( $\beta$-Galsil) que en forma general causa un deterioro neurológico progresivo, regresión motora, hipertonía muscular y espasticidad, convulsiones, irritabilidad, pérdida de la audición y en un $85 \%$ de los casos la muerte temprana. La enzima $\beta$-Galsil cataliza la hidrólisis de galactoceramida en galactosa y ceramida (N-Acilesfingosina), así como la deacilación de derivados galactosilesfingosina (psicosina) (Figura 1) y requiere como cofactor para el proceso la Saposina A cuya alteración también puede generar la enfermedad (3).
El bloqueo en la actividad catalítica, causa un proceso acumulativo de galactoceramida y galactosilesfingosina altamente tóxico para el microambiente celular, debido a que produce una desaparición rápida de la mielina que cubre los axones (sustancia blanca) y la destrucción de las células formadoras de mielina (oligodendrocitos) en el Sistema Nervioso Central (SNC), como también de las células de Schwann o células gliales en el Sistema Nervioso Periférico (SNP) $(4,5)$. La lesión en los oligodendrocitos produce una respuesta en las células gliales llamada "gliosis reactiva" que ocasiona un cambio en la morfología de los astrocitos al aumentarlos de tamaño y pasándolos a una forma "fibrosa-reactiva". También la galactosilceramida puede infiltrar a los macrófagos formando las "células globoides", característicos de la enfermedad (6).

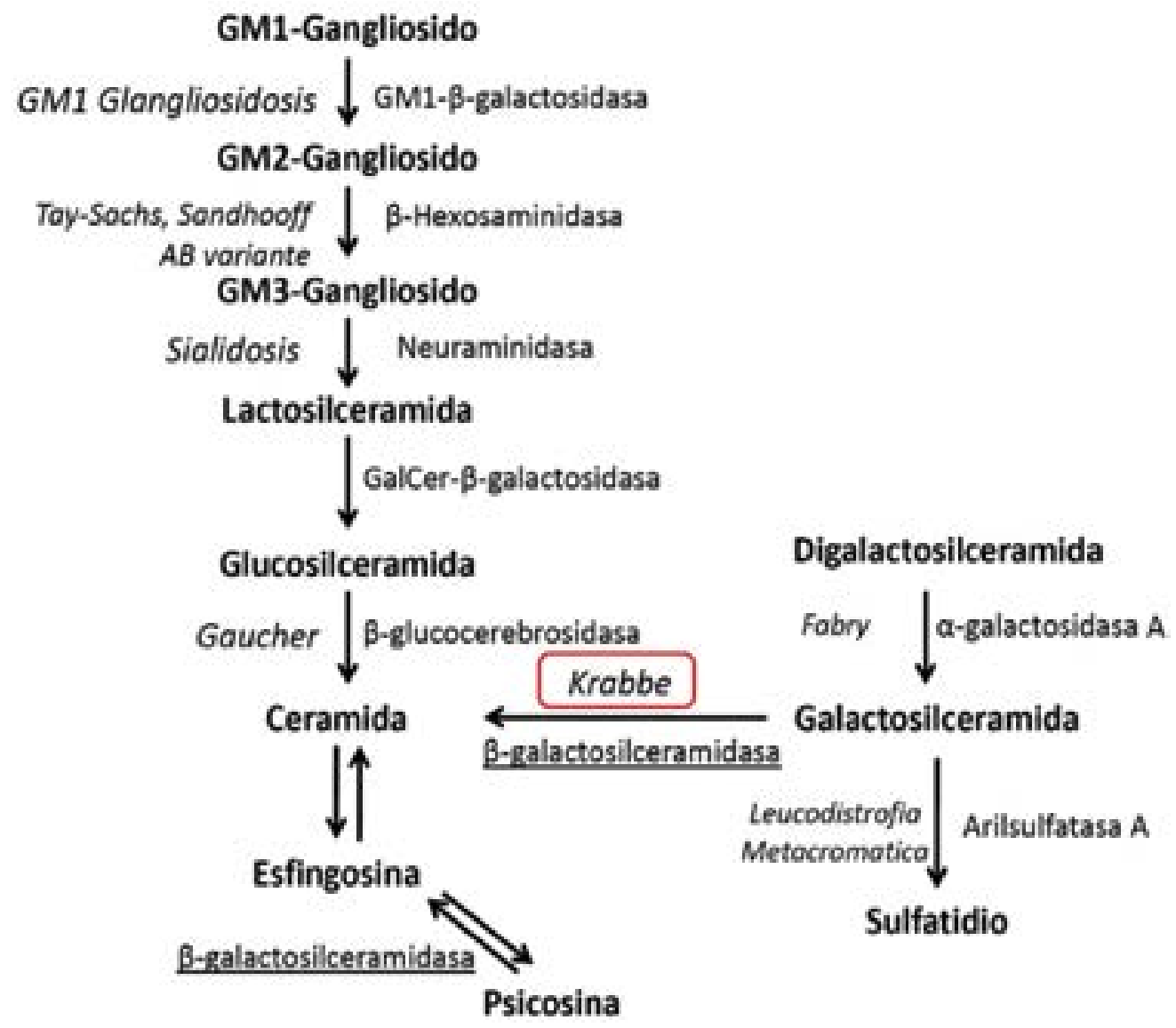

Figura 1. Ruta metabólica de degradación de esfingolípidos. Se observan las vías metabólicas donde participa la enzima $\beta$-Galsil en la formación de ceramida a partir de galactosilceramida y de esfingosina a partir de psicosina. Tomado y modificado de King W.,2009 (7). 
En relación a los aspectos moleculares la $\beta$-Galsil es codificada por el gen GALC (GALC; MIM\# 606890) (8) que se encuentra ubicado en el cromosoma 14 en la posición q24.3-q32.1 (9) y tiene un tamaño de 3795 pb, está formado por 17 exones y 16 intrones (10). La enzima está constituida por 669 aminoácidos con seis sitios potenciales de glucosilación en el aminoácido asparagina (11). La proteína precursora tiene un tamaño entre $80-85 \mathrm{kD}$ y está conformada por dos subunidades de $30 \mathrm{kD}$ y otra de $50-52 \mathrm{kD}$. (12). El gen GALC presenta una amplia variedad de mutaciones y polimorfismos entre las cuales se encuentran la deleción de $30 \mathrm{~kb}$ ubicada en la parte media del intrón 10 (IVS10del 30kb), esta mutación se ha observado en el $45 \%$ de los pacientes afectados con enfermedad de Krabbe de ascendencia europea y se asocia con el fenotipo de aparición temprana, mientras que la mutación 809G>A está asociada con la aparición tardía de la entidad $(3,13)$.

Los aspectos clínicos de la enfermedad de Krabbe abarcan un espectro amplio de expresiones fenotípicas; se documentan casos de aparición temprana caracterizada por un deterioro neurológico progresivo que genera la muerte antes de los dos años de edad; o de inicio tardío, a finales de la niñez, o principio de la adolescencia o adultez, descrita con una lenta progresión de la enfermedad, con pérdida de habilidades adquiridas (14).

En la forma infantil el cuadro clínico presenta tres estadios. El primer estadio se observa irritabilidad, rigidez, retraso en el desarrollo mental y motor, fiebre sin infección, hipersensibilidad auditiva, táctil y visual, llanto frecuente sin causa aparente, vómitos y otros problemas de alimentación pueden dar lugar a la pérdida progresiva de peso. El segundo estadio se caracteriza por una rápida y severo deterioro psicomotor, hipertonicidad, y atrofia óptica, en el último estadio el niño es ciego, sordo y el daño neurológico catastrófico (14).

En las formas de inicio tardío, los fenotipos pueden diferir considerablemente entre los individuos, pero se ha observado pérdida de la visión con hemiparesia, ataxia y regresión psicomotora. La mayoría de los niños con la forma juvenil mostraron un deterioro rápido inicial seguido de una progresión más gradual que dura por años $(14,15)$. Siguiendo este último aspecto, un estudio en New York con 122 pacientes con EK encontró que 62\% presentaban una manifestación infantil temprana, $10 \%$ entre los 7 a 10 meses, $22 \%$ de 13 meses a 10 años y un $5 \%$ entre la etapa de la adolescencia y adultez (15).
Con respecto a la incidencia de la EK, se ha reportado para Estados Unidos y Europa una frecuencia de 1:100000 recién nacidos, pero estudios recientes han demostrado valores mayores de 1:22.000 aproximadamente en New York (16). En América Latina no se conoce la incidencia real de esta enfermedad, ni estudios que reporten valores de referencia de actividad para la enzima afectada, sin embargo se ha documentado un reporte de caso en México donde se realizó el diagnostico de un paciente EK por microscopia transmisión electrónica (17) y un tamizaje de alto riesgo en Brasil, para 10,000 individuos con sospecha de un error innato del metabolismo y donde se hallaron 13 pacientes con deficiencia de $\beta$-Galsil (18).

La situación en Colombia es más compleja, dado que estos desordenes no son estudiados bajo programas de pesquisa estatal, sino estrictamente bajo sospecha clínica, lo que conlleva a diagnósticos tardíos, dando lugar a que un buen número de individuos mueran sin el beneficio del diagnóstico y sin que las familias afectadas tengan la posibilidad de una asesoría genética adecuada.

Se presenta entonces a la comunidad científica un estudio de valores de referencia en población control colombiana para la actividad leucocitaria de $\beta$-Galsil usando dos protocolos de valoración enzimática y la aplicación de dicha metodología estandarizada a una población de alto riesgo para enfermedad neurodegenerativa de posible etiología lisosomal.

\section{Materiales y métodos}

\section{Muestreo}

Esta investigación está acorde a la resolución 8430 de 1993 del Ministerio de Salud de Colombia e involucro un total de 259 muestras (Periodo: 2010 -2016), de las cuales 110 fueron individuos Control y 149 pacientes con Compromiso Neurodegenerativo (CND), de estos últimos en el $63 \%(n=94)$ se reportó al laboratorio compromiso de la sustancia blanca, valorado por resonancia magnética nuclear y en un $20 \%(n=30)$ se informó estudios de velocidades de conducción nerviosa anormal (Lento). En el $17 \%$ restante de las remisiones $(n=25)$ no se recibió información relevante para la enfermedad en estudio. Las edades de controles y pacientes fueron de 3 meses a 64 años, sin embargo, el $80 \%$ de la población en tamizaje para EK eran menores de 10 años.

Los especímenes recolectados fueron utilizados para establecer la actividad enzimática de la $\beta$-Gasil por dos mé- 
todos de punto final (colorimétrico y fluorométrico). Los análisis fueron ordenados por médicos pediatras, neurólogos y neuropediatras de diversas regiones del país al Centro de Investigaciones en Bioquímica, de la Universidad de los Andes.

\section{Muestras}

Los estudios enzimáticos involucraron de 8 a $10 \mathrm{ml}$ de sangre total en tubos que fueron recolectados en $2 \mathrm{ml}$ de dextran-heparina. Los procedimientos de aislamiento y lisis leucocitaria, siguieron los procedimientos reportados por Shapira et al 1989 (19). La valoración proteica de lisado leucocitario fue llevado a cabo mediante el método BCA Protein Assay Kit Thermo Scientific No. 23225 (20).

\section{Actividad Enzimática de $\beta$-Galsil por el método colorimétrico}

\section{Preparación de sustrato}

Para la medición de la actividad de $\beta$-Galsil por el método colorimétrico se uso el sustrato ácido $\omega-2,4,6$ - trinitrofenil aminolaúrico galactosil esfingosina (TNPAL-Galactocerebrosida Sigma T0764), el cual fue elaborado utilizando el método reportado por Besley et al, 1981 a partir de $5 \mathrm{mg}$ de TNPAL-galactocerebrosido en $2,5 \mathrm{ml}$ de solución cloroformo-metanol 2:1, este fue mezclado con $5 \mathrm{ml}$ taurocolato de sodio $5 \mathrm{~g} / \mathrm{L}$ (Sigma T0875) y $300 \mu \mathrm{l}$ ácido Oleico $10 \mathrm{~g} / \mathrm{L}$ (Sigma O1008). Se elaboraron alícuotas $165 \mu \mathrm{l}$ en tubos eppendorf de $1.5 \mathrm{ml} \mathrm{y}$ se llevaron a evaporación a $37^{\circ} \mathrm{C}$ con corriente de nitrógeno gaseoso, el sustrato final fue almacenado a $-20{ }^{\circ} \mathrm{C}$. En el momento de realizar el procedimiento analítico, el sustrato TNPAL-galactocerebrosido fue resuspendido en $210 \mu \mathrm{l}$ de buffer de citrato-fosfato $0,5 \mathrm{~mol} / \mathrm{L} \mathrm{pH} \mathrm{4,5}$.

\section{Actividad enzimática}

El producto de la lisis del extracto leucocitario se diluyó a una concentración final de proteína $\geq 60 \mathrm{mg} / \mathrm{dL}$ en un volumen final de $180 \mu \mathrm{L}$ en un tubo de $0.5 \mathrm{ml}$. De ésta dilución se tomaron $50 \mu \mathrm{L}$ y se adicionó por duplicado a tubos de $1.5 \mathrm{ml}$ y luego se agregaron 40 $\mu \mathrm{l}$ de sustrato TNPAL-Galactocerebrosido $0,74 \mathrm{mM}$ en forma respectiva, el blanco de reactivo fue procesado de igual manera excepto que no contenía muestra, esta fue adicionada después de agregar la solución de parada. Todos los tubos fueron incubados por 18 horas a $37^{\circ} \mathrm{C}$. Terminada la reacción se agregó de forma secuencial:
$750 \mu \mathrm{l}$ de Isopropanol/heptano/ $\mathrm{H}_{2} \mathrm{SO}_{4} 1 \mathrm{~N} \quad(40 / 10 / 1$ $\mathrm{v} / \mathrm{v} / \mathrm{v}), 450 \mu \mathrm{l}$ Heptano y $400 \mu \mathrm{l}$ agua destilada ultrapura, la mezcla se agitó suavemente mediante vortex y se centrifugó a 1000 g o 3500 rpm por 5 min. Finalmente de éste último sobrenadante se tomó $600 \mu \mathrm{l}$ y se leyó la absorbancia a $330 \mathrm{~nm}$ en un espectrofotómetro Biomate 3 (21,22).

\section{Actividad Enzimática de $\beta$-Galsil por el método fluorométrico}

\section{Preparación de sustrato}

En este método se usó como sustrato 6-Hexadecanoil amino 4-metilumbeliferil $\beta$-D-galactosido (HMU- $\beta G a l) ~ d e$ Moscerdam Substrates, el cual fue elaborado a partir de 7,5 mg de HMU- $\beta$ Gal en $14,1 \mathrm{ml}$ de solución cloroformo-metanol 2:1, este fue mezclado con $3,18 \mathrm{ml}$ taurocolato de sodio $30 \mathrm{~g} / \mathrm{L}$ y $4,75 \mathrm{ml}$ ácido oleico $6 \mathrm{~g} / \mathrm{L}$. Se elaboraron alícuotas del sustrato de $210 \mu \mathrm{l}$, luego cada tubo se evaporó a sequedad a $37{ }^{\circ} \mathrm{C}$ con corriente de nitrógeno gaseoso y fueron almacenados a $-20{ }^{\circ} \mathrm{C}$ hasta su uso.

Para el procedimiento de la determinación de actividad enzimática, el sustrato HMU- $\beta$ Gal previamente llevado a sequedad fue resuspendido con $210 \mu \mathrm{l}$ de Buffer de citrato-fosfato $0,25 \mathrm{~mol} / \mathrm{L} \mathrm{pH} \mathrm{5,2.}$

\section{Actividad enzimática}

El extracto de leucocitos sometido a lisis fue ajustado a una concentración de proteína $\geq 10 \mathrm{mg} / \mathrm{dL}$ en un volumen final de $120 \mu \mathrm{L}$. De éste último se tomaron por duplicado $50 \mu \mathrm{l}$ muestra y a cada uno de los tubos se le adicionó 21 $\mu \mathrm{l}$ de sustrato HMU- $\beta$ Gal 0,040 mM, llevando a incubación por 18 horas a $37^{\circ} \mathrm{C}$. El blanco fue tratado de igual manera excepto que la enzima fue agregada después de parada la reacción. Finalizada la incubación a la reacción se le agrego $650 \mu \mathrm{l}$ Glicina-carbonato $\mathrm{pH} 10$ para detener el proceso, ésta última mezcla fue pasada a tubos de borosilicato y se midió su fluorescencia a $360 \mathrm{~nm}$ excitación y $450 \mathrm{~nm}$ emisión en un Fluorómetro SequoiaTurner 450 $(21,23)$. (Figura 2) Como enzima control se realizó un ensayo enzimático para la $\beta$-Galactosidasa cuya deficiencia está relacionada con la enfermedad Mucopolisacaridosis tipo IVB y Gangliosidosis $\mathrm{GM}_{1}$, para lo cual se utilizó la técnica fluorométrica reportada por Shapira (24) y Modificada por Uribe y colbs $(25,26)$. Curva de calibración con 4-Methylumbelliferona (4-MU)* * 


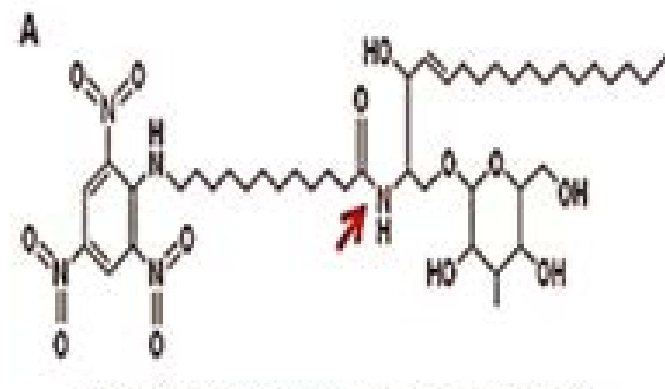

Trinitrofenilaminolaurol-galactocerebrosido

(TNPAL-Galactocerebrosido)

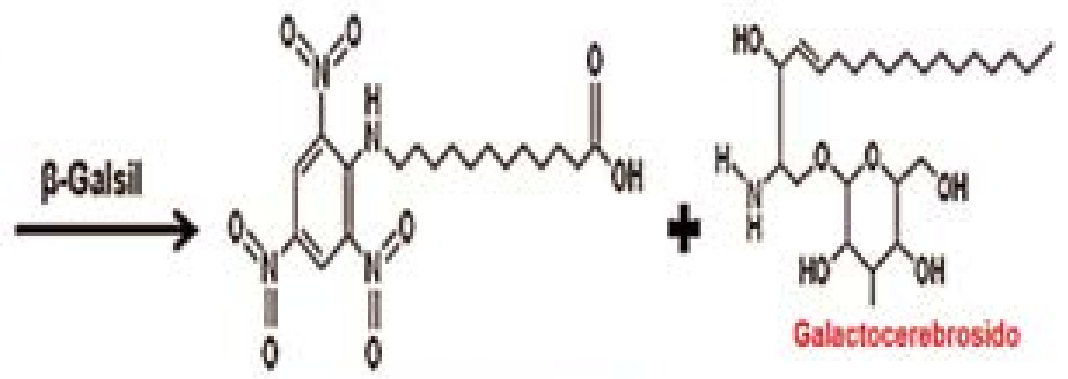

Acido trinitrofenilaminolaurico
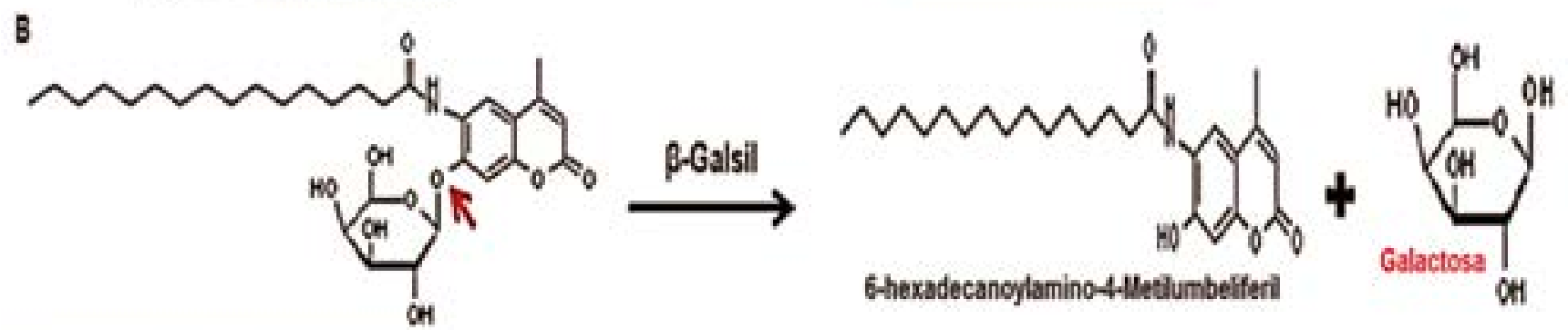

6-Hexadecanoylamino-4tlatilumbeliferi-

Beta-D-Galactosido

(AMU.BGal)

Figura 2. Reacciones de los sustratos frente a la $\beta$-Galsil. A. El sustrato TNPAL-Galactocerebrosido es hidrolizado en Galactocerebrosido de color amarillo y ácido trinitrofenilaminolaurico cuya magnitud máxima de absorción a los 330nm. B. El Sustrato HMU-BGal, que se hidroliza en galactosa y 6-hexadecanoylamino-4-Metilumbeliferil un marcador fluorogénico (Excitación Máxima: 360 nm - Emisión Máxima: 450 nm)

Curva de calibración con 4-Methylumbelliferona $(4-M U)^{* *}$

A partir de un estándar de 4-MU (22,5 mg /10 ml Glicina-carbonato $0,17 \mathrm{M} \mathrm{pH} 10$, se elaboró una curva de calibración con un rango de concentraciones entre $5.675-0.011 \mathrm{nmol} / 100 \mu \mathrm{l}$ para la determinación de la actividad enzimática de $\beta$-Galactosidasa y $\beta$-Galsil. (** Material provisto por Sigma (St. Louis, MO, USA).

\section{Análisis estadístico}

La estadística descriptiva y representación gráfica de los datos fue realizada mediante el paquete estadístico Statistix® 9 provisto por Analytical Software. Se aplicó la prueba no paramétrica Shapiro-Wilk para valorar la distribución de los grupos control. El punto de corte entre deficientes y controles fue establecido mediante el análisis de Curvas ROC (Receiver operating Characteristics) $(27,28)$.

\section{Resultados}

Para las 110 muestras analizadas del grupo control se halló un rango de actividad en la enzima $\beta$-Galsil de 2.04 - 9,28 nmol/mgprot/h (Media: 3,86) por el método colorimétrico y de 0,3 - 3,28 nmol/mgprot/h (Media: $1,14)$ en el método fluorométrico. En el grupo en remisión $(n=149)$, se encontró un paciente con EK con actividades de $1,85 \mathrm{nmol} / \mathrm{mgprot} / \mathrm{h}$ por la técnica colorimétrica y de $0,030 \mathrm{nmol} / \mathrm{mgprot} / \mathrm{h}$ por la técnica fluorométrica, hallazgos compatibles con enfermedad de Krabbe. (Tabla 1 y Figura 3). 
Tabla 1. Valores de Referencia de $\beta$-Galactosilceramidasa * *

**El análisis descriptivo se hizo en el programa Statistix $® 9,{ }^{1}$ Afectados Infantiles y ${ }^{2}$ Afectados Adultos

\begin{tabular}{|c|c|c|c|c|c|c|c|c|}
\hline \multirow{2}{*}{ TECNICA } & \multirow{2}{*}{ EDAD } & \multicolumn{6}{|c|}{ Actividad Enzimatica en nmolmg proteinahora } & \multirow{2}{*}{ Referencia } \\
\hline & & Muestra & Mrima & Máxima & Media & so & Literatura & \\
\hline \multirow{3}{*}{ Colorimétrica } & 3 meses - 64 ahlos & Controles & 2,04 & 9,28 & 3,86 & 1,47 & \multirow[b]{2}{*}{$3,70-10,33$} & \multirow{3}{*}{$\begin{array}{c}\text { Svennerholm, } \\
1981\end{array}$} \\
\hline & 3 meses -57 aftos & CND pocents & 2,04 & 14,9 & 4,35 & 2,54 & & \\
\hline & 9 años & Afectado & - & 1,85 & - & - & $0,0-1,172$ & \\
\hline \multirow{3}{*}{ Fluorométrica } & 3 meses - 64 ahlos & Controles & 0,3 & 3,28 & 1,14 & 0,65 & \multirow[b]{2}{*}{$1,05-4,41$} & \multirow{3}{*}{$\begin{array}{c}\text { Wiederschain } \\
1992\end{array}$} \\
\hline & 3 meses -57 ahlos & CND wacesen & 0,28 & 4,21 & 1,20 & 0,78 & & \\
\hline & 9 afios & Afectado & - & 0,03 & - & - & $\begin{array}{c}0,0-0,02^{\prime \prime} \\
0,13-0,20^{2}\end{array}$ & \\
\hline
\end{tabular}
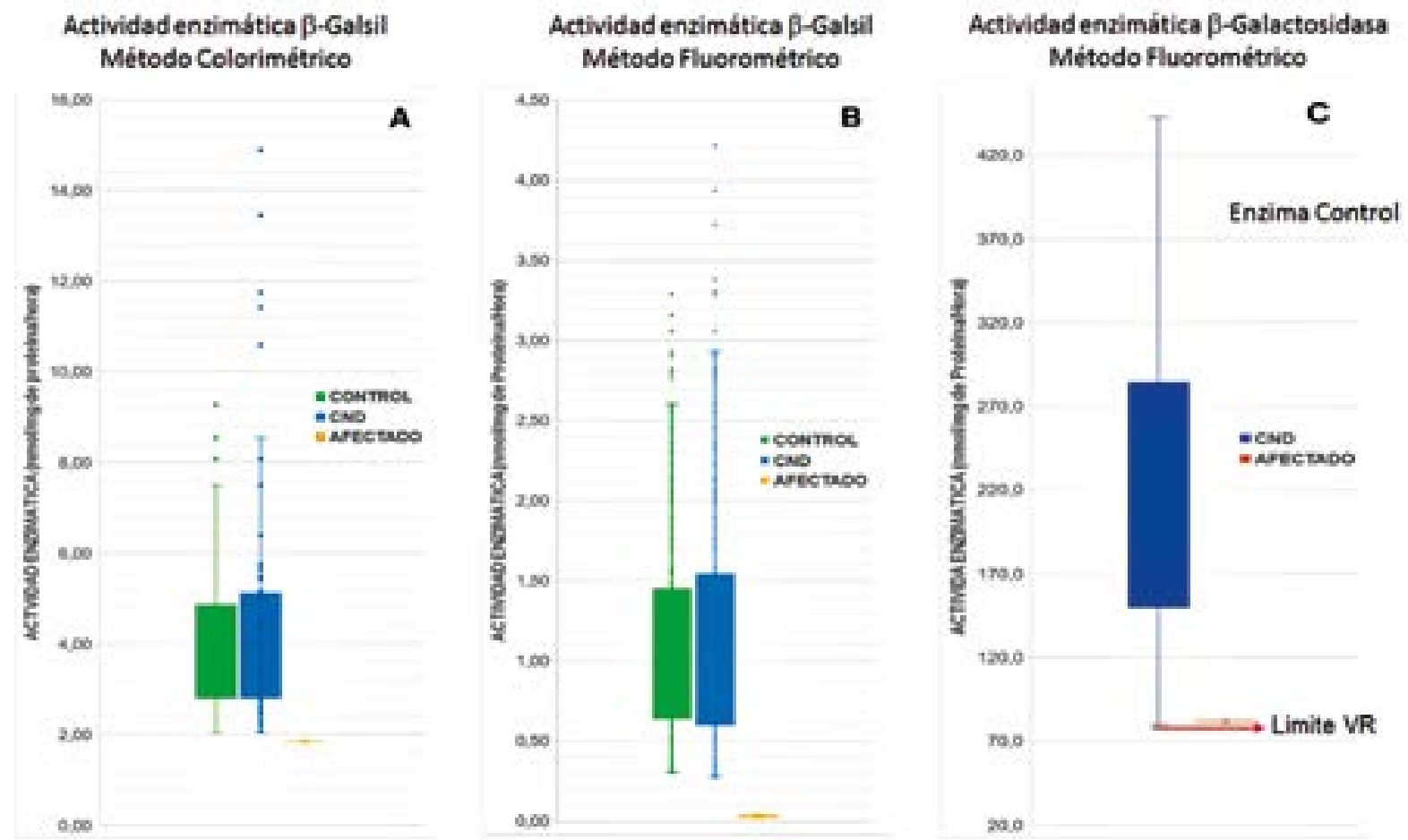

Figura 3. Actividad Enzimática de $\beta$-Galsil. Graficas A/B. Se Observa el comportamiento de la actividad de la $\beta$-Gasil en Población control y pacientes remitido por compromiso Neurodegenerativo, contrastando con el único paciente con deficiencia para la Enzima. Grafica C. Representa la actividad de la enzima $\beta$-Galactosidasa de grupo CND, que se encuentra dentro de limite de referencia para Colombia (80 -557 nmol/mg proteina/Hora). 
A manera de control de procedimiento experimental, el paciente con actividad deficiente para $\beta$-Galsil $(0,034$ $\mathrm{nmol} / \mathrm{mgprot} / \mathrm{h}$ ), fue valorado para la enzima Arilsufatasa A (ASA), una proteína catalítica involucrada en el metabolismo de los esfingolípidos cuya deficiencia está relacionada a otra enfermedad degenerativa de sustancia blanca, documentada como Leucodistrofia Metacromática (Figura 1). Los resultados obtenidos son normales para este agente enzimático y consistentemente deficientes para la alteración metabólica en estudio. (Tabla 2).
Con el fin de garantizar la calidad de todas las muestras procesadas (Casos y Controles) se cuantifico la actividad lisosomal de la $\beta$-galactosidasa, encontrándose 146 pacientes dentro del limite de referencia reportado para Colombia, $80-557 \mathrm{nmol} / \mathrm{mgprot} / \mathrm{h}(26,29)$, tres casos mostraron deficiencia de causa genética de la enzima control, no incluidos en la graficación. Para las curvas de calibración de 4MU se obtuvo un coeficiente de correlación $\left(\mathrm{r}^{2}\right)$ de 0,997 con un coeficiente de variabilidad de $1,8 \%$.

\begin{tabular}{|c|c|c|c|c|}
\cline { 2 - 5 } \multicolumn{1}{c|}{} & \multicolumn{2}{c|}{ Enzimas Control } & \multicolumn{2}{c|}{$\begin{array}{c}\text { Enzima } \beta \text {-Galactosilceramidasa } \\
\mathrm{nmol} / \mathrm{mg} \text { prot/h }\end{array}$} \\
\cline { 2 - 5 } & $\begin{array}{c}\beta \text {-Galactosidasa } \\
\text { (Fluorométrica) } \\
\mathrm{nmol} / \mathrm{mg} \mathrm{prot} / \mathrm{h}\end{array}$ & $\begin{array}{c}\text { Arilsulfatasa A } \\
\text { (Colorimétrica) } \\
\mathrm{nmol} / \mathrm{mg} \text { prot/h }\end{array}$ & Colorimétrica & Fluorométrica \\
\hline $\begin{array}{c}\text { Valores } \\
\text { de referencia }\end{array}$ & $\mathbf{8 0 - 5 5 7}$ & $\geq \mathbf{1 0 0}$ & $\mathbf{2 . 0 2 - 1 4 . 9 3}$ & $\mathbf{0 , 3 0 - 3 , 9 3}$ \\
\hline Paciente con EK & $\mathbf{8 2 , 0}$ & $\mathbf{1 0 6 , 7}$ & $\mathbf{1 , 8 5}$ & $\mathbf{0 , 0 3 4}$ \\
\hline
\end{tabular}

Tabla 2. Actividad del Paciente con baja actividad de $\beta$-Galsil

\section{Discusión y conclusiones}

El tamizaje involucró 149 pacientes que evidenciaron un cuadro de regresión neurológica y compromiso de sustancia blanca fundamentalmente, el $80 \%$ de las remisiones se orientaban a la búsqueda de formas infantiles tempranas o tardías de esfingolipidosis (Ver Figura 4).

A partir de este grupo de riesgo, se logró identificar un individuo afectado con Enfermedad de Krabbe que expresa una actividad residual para $\beta$-Galsil de $3 \%$, valor calculado a partir de la mediana de los valores para población control del método fluorescente. En contraste la técnica colorimétrica no fue discriminante debido a que el valor de actividad es muy cercano al límite inferior del valor de referencia (Tabla 2) y adicionalmente se encontraron falsos positivos, relacionados a individuos afectados por otras enfermedades de depósito lisosomal (v. gr. Enfermedad de Gaucher) (Tabla 3).
El estudio permitió establecer un valor preliminar de prevalencia de 1/149 en la población analizada para enfermedad de Krabbe, sin embargo, es necesario ampliar el tamaño de la muestra para establecer la verdadera frecuencia de esta enfermedad en Colombia.

Con respecto a los valores de referencia encontrados para las 110 muestras control en la técnica colorimétrica se ubicó de 2.04 a $9.28 \mathrm{nmol} / \mathrm{mgprot} / \mathrm{h}$ en leucocitos, los cuales presentaron ligeras diferencias de actividad con relación al estudio realizado por Svennerholm en 1981 en Suecia (30), el cual reportó actividades en un rango de $3.7-10.33 \mathrm{nmol} / \mathrm{mgprot} / \mathrm{h}$, esto se debe a que se usó un sustrato colorimétrico y una muestra poblacional diferente que explica las variaciones en la actividad enzimática. En comparación la técnica fluorométrica presentó una concordancia con los valores reportados por Wiederschain en 1992 (Tabla 1) (23). 


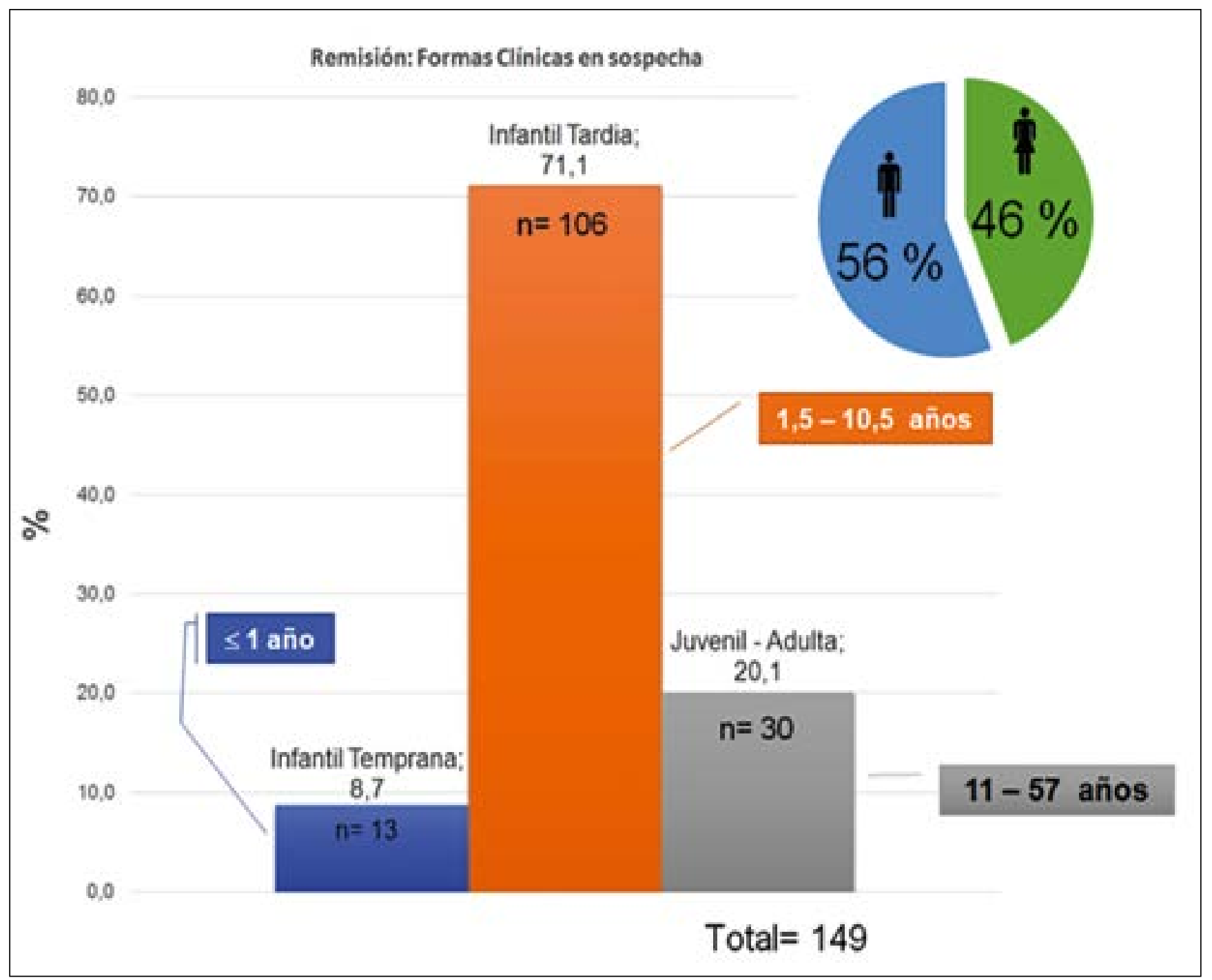

Figura 4. Población remitida a estudio para ß-Galactosilceramidasa (\%). Se observa la distribución de la población remitida a estudio agrupada en grupos de edad que se asocian a fenotipos reportados de la enfermedad de Krabbe.

Un hallazgo de gran relevancia se encontró al realizar un experimento alterno, que consistió en incluir individuos con sospecha de enfermedad de Gaucher $(n=13)$ con el fin de explorar el comportamiento enzimático de pacientes afectados en la misma ruta metabólica de los esfingolípidos donde opera $\beta$ Galsil, usando el sustrato TNPAL-galactosilcerebrosido (método colorimétrico). Los resultados mostraron una baja actividad para la enzima en 4 muestras donde se esperaba una actividad normal. (Tabla 3) De estos individuos tres fueron diagnosticados con la enfermedad de Gaucher al demostrase la deficiencia de $\beta$-glucosidasa, mientras que la actividad $\beta$-Galsil con substrato fluorométrico fue normal. Este último resultado permite proponer que el substrato colorimétrico no es especifico y que la actividad obtenida puede ser debida a que está involucrada más de una enzima de la ruta de los esfingolípidos, aspecto que explicaría por qué en el caso detectado para EK no se evidenció mayor diferencia entre el paciente y los controles normales. 
Tabla 3. Comparación resultados entre $\beta$-Galsil (técnica colorimétrica) y resultados pacientes en estudio para $\beta$-Glucosidasa.

\begin{tabular}{|c|c|c|c|}
\hline Individuos & $\begin{array}{c}\text { Técnica Colorimétrica (nmol/mgprot/hora) } \\
\text { VR: } 2,04-9,28\end{array}$ & $\begin{array}{c}\text { Resultados } \\
\beta \text {-Galsil }\end{array}$ & $\begin{array}{c}\text { Resultados } \\
\beta \text {-Glucosidasa ** }\end{array}$ \\
\hline 1 & 2,99 & Normal & Normal \\
\hline 2 & 3,28 & Normal & Normal \\
\hline 3 & 0,53 & ¿Afectado? & Afectado $^{1}$ \\
\hline 4 & 5,39 & Normal & Normal \\
\hline 5 & 2,89 & Normal & Normal \\
\hline 6 & 3,00 & Normal & Normal \\
\hline 7 & 0,81 & ¿Afectado? & Afectado $^{1}$ \\
\hline 8 & 1,26 & ¿Afectado? & Afectado $^{1}$ \\
\hline 9 & 1,55 & Afectado *** & Normal \\
\hline 10 & 4,39 & Normal & Afectado $^{1}$ \\
\hline 11 & 2,33 & Normal & Normal \\
\hline 12 & 3,02 & Normal & Normal \\
\hline 13 & 2,08 & Normal & Afectado $^{1}$ \\
\hline
\end{tabular}

** La $\beta$-Glucosidasa se encuentra deficiente en la enfermedad de Gaucher y pertenece a la ruta metabólica de los esfingolípidos. ${ }^{1}$ Paciente con deficiencia de $\beta$-Glucosidasa.

*** Paciente con hallazgos sugerentes de Enfermedad de Niemann-Pick.

Otra observación importante encontrada dentro del grupo de pacientes tamizados, está relacionada a la detección de otros desordenes del metabolismo de los esfingolípidos, en razón a que las enzimas control utilizadas pertenecen también a la secuencia metabólica en la que participa $\beta$-Galsil y cuya deficiencia se relaciona a cuadros clínicos neurodegenerativos. Bajo este aspecto el estudio evidenció 2 casos de Gangliosidosis GM1 (Deficiencia de $\beta$-Galactosidasa) y 4 casos de Leucodistrofia Metacromática (Deficiencia de Arilsufatasa A).

Sin embargo, es conveniente indicar la sospecha tardía de estas anormalidades metabólicas, dado que menos del $9 \%$ de la población de pacientes remitido correspondía a variantes de presentación temprana, de hecho el caso detectado corresponde a un infante de sexo masculino de 9 años de edad, producto de primer embarazo, cuyo diagnostico enzimático se hace en la fase terminal de la enfermedad, un hallazgo que no difiere de las otras alteraciones de curso degenerativo detectadas por el estudio $(\mathrm{n}=6)$, cuyos pacientes estaban en un rango de 0,5 a 30 años (El registro, solo muestra un caso con sospecha clínica temprana que es diagnosticado a los seis meses de vida) (31) (Tabla 4).
Finalmente, en lo referente a las metodologías analíticas los resultados muestran que el método fluorométrico tiene una alta especificidad para la valoración de la actividad de $\beta$-Galsil, lo que es adecuado para la implementación de proyectos futuros de tamizaje de alto riesgo en individuos que presentan compromiso neurodegenerativo de sustancia blanca, aspecto que ofrece la posibilidad de un diagnóstico temprano de la enfermedad de Krabbe en Colombia, hecho que permitirá acceder a un manejo médico oportuno mejorando la calidad de vida del paciente y de la familia.

Este último aspecto será muy importante a futuro, dado que se ha reportado que el trasplante de medula ósea o de células madre hematopoyéticas, puede retrasar el curso natural de la enfermedad en casos de presentaciones infantiles tempranas con detección precoz o formas adultas leves (32). Igualmente, se ha documentado el desarrollo de la terapia de reemplazo enzimático que en su etapa pre-clínica, ha mostrado resultados muy alentadores en lo referente a la mielinización de cerebro y medula espinal, al igual que la experimentación en modelos animales con terapia de reducción de substrato que podrían ser aplicados a mediano plazo. (33 - 36). 
Tabla 4. Pacientes con otros desordenes neurodegenerativos de causa metabólica detectados en el tamizaje.

Actividad enzimática (nmol/mg de Proteína/Hora)

\begin{tabular}{c|c|c|c|c}
\hline Enzima & Arilsulfatasa A & $\beta$-Galactosidasa & $\beta$-Galsil * & Hexosaminidasa Total \\
\hline Valor de Referencia & $\mathbf{1 0 0}$ & $\mathbf{8 0 - 5 5 7}$ & $\mathbf{0 , 3 0}-\mathbf{3 , 9 3}$ & $\mathbf{6 8 0}-\mathbf{2 1 0 0}{ }^{* *}$ \\
\hline CND $^{\text {Paciente(Edad) }}$ & \multicolumn{4}{|l}{} \\
\hline 12286 (30 años) & 6,6 & 305,8 & 1,20 & 751,2 \\
\hline 17344 (6 años) & 12,8 & 183,8 & 1,40 & 1058,5 \\
\hline 12112 (2,5 años) & 117,3 & 3,2 & 1,13 & 1468,1 \\
\hline 14900 (1,5 años) & 138,4 & 3,5 & 0,84 & 1029,4 \\
\hline 10881 (5 años) & 2,6 & 304,2 & 0,82 & 763,9 \\
\hline 21026 (0,5 años) & 32,1 & 138,1 & 1,70 & 722,8 \\
\hline
\end{tabular}

* Técnica Fluorométrica

** Se evalúa Hexosaminidasa total como otra enzima control para descartar que los resultados para b-Galactosidasa sean causados por deterioro de la muestra. El método corresponde a técnica fluorométrica reportada por Shapira (24) y los Valores de Referencia corresponden al estudio de Argumedo, N., \& Uribe, J. A., 2012. (31)

\section{Conflicto de Intereses}

Los autores del presente trabajo declaran no tener ningún tipo de conflicto de intereses que invalide la presente investigación

\section{Agradecimientos}

Los autores desean expresar su agradecimiento a las $\mathrm{Di}$ rectivas de la Facultad de Ciencias y el Departamento de Ciencias Biológicas de la Universidad de los Andes, por el apoyo brindado a través de los Proyectos Semilla, para la viabilidad de esta investigación.

\section{Referencias}

1. Schomburg I, Chang A, Schomburg D. Brenda, enzyme data and metabolic information. Nucleic Acids Res., 2002; (30):47-9

2. Scheer M, Grote A, Chang A, Schomburg I, Munaretto C, Rother M, Sóhngen C, Stelzer M, Thiele J, Schomburg D. Brenda, the enzyme information system in 2011. Nucleic Acids Res., 2011; (39):670-76.

3. Wenger, D. A., Suzuki, K., Suzuki, Y., and Suzuki, K. Galactosylceramide lipidosis: Globoid cell leukodystrophy
(Krabbe disease). En: Valle D, Beaudet A, Vogelstein B, Kinzler K, Antonarakis SE, Ballabio An Editors the Metabolic and Molecular Basic of Inherited Disease 8th New York: McGraw-Hill, 2001, p. 3669 - 3694.

4. Barone R, Brühl K, Stoeter P, Fiumara A, Pavone L, Beck M. Clinical and Neuroradiological Findings in Classic Infantile and Late-Onset Globoid-Cell Leukodystrophy (Krabbe Disease). Am J Med Genet., 1996; (63):209-11.

5. Giri S, Khan M, Rattan R, Singh I, Singh AK. Krabbe disease: psychosine-mediated activation of phospholipase A2 in oligodendrocyte cell death. J Lipid Res., 2006; (47): 1478 - 92.

6. Suzuki K, Suzuki Y. Globoid Cell Leucodystrophy (Krabbe's Disease). Deficiency of Galactocerebroside B-Galactosidase. Proc Natll Acad Sci., 1970; (66):302-9.

7. King MW. The Medical Biochemistry [on line] 2012. Avialable in http://themedicalbiochemistrypage.org/index. php

8. Tappino B, Biacheri R, Mort M, Regis S, Corsolini F, Rossi A, Stroppiano M, Lualdi S, Fiumara A, Bembi B, Di Rocco M, Cooper D, Filocamo M. Identification and characterization of 15 Novel GALC gene mutations causing Krabbe Disease. Hum Mutat., 2010; (31): E1894-E1915.

9. Oehimann, Ralph, Zlotogora, Joel, Wenger, David A., Knowlton, Robert G Localization of the Krabbe Disease 
Gene (GALC) on chromosome 14 by Multipoint linkage analysis. Am J Hum Genet., 1993; (53):1250-55.

10. Luzi P, Rafi MA, Wenger DA. Structure and organization of the human galactocerebrosidase (GALC) gene. Genomics., 1995; (26);407-09.

11. Sakai N, Inui K, Fujii N, Fukushima H, Nishimoto J, Yanagihara I, Isegawa Y, Iwamatsu A, Okada S. Krabbe disease: isolation and characterization of a full-length cDNA for human galactocerebrosidase. Biochem Biophys Res Commun., 1994; (198):485-91.

12. Chen YQ, Rafi MA, De Gala G, Wenger DA. Cloning and expression of cDNA encoding human galactocerebrosidase, the enzyme deficient in globoid cell leukodystrophy. Hum Mol Genet., 1993; (2):1841-45.

13. Luzi P, Rafi MA, Wenger DA. Multiple mutations in the GALC gene in a patient with adult-onset Krabbe disease. Ann Neurol., 1996; (40):116-19.

14. Wenger D. Krabbe Disease En Associate editors Pagon RA, Bird TD, Dolan C, Stephens K, and Adam M. GeneReviews [on line] University of Washington (WA) Seattle, 2011. Avialable in www.ncbi.nlm.nih.gov

15. Duffner PK, Barczykowski A, Kay D, Jalal K, Yan L, Abdelhalim A, Gill S, Gill AL, Carter R. Later Onset Phenotypes of Krabbe Disease: Results of the World-Wide Registry. Pediatric Neurology., 2012; (46):298 - 306.

16. Duffner PK, Caggana M, Orsini J, Wenger D, Patterson MC, Crosley CJ, y Others. Newborn Screening for Krabbe Disease: the New York State Model. Pediatr Neurol., 2009; (40):245-52.

17. Villegas H, Hernández A, Peralta S. Vásquez y Reyes B. Diagnóstico de leucodistrofia de Krabbe por microscopia electrónica de transmisión. Informe de un paciente. Cir Ciruj., 2006; (74):477-81.

18. Coelho J.C., Waner M., Burin M.G., Vargas C.R., Giugliani R. Selective screening of 10.000 high-risk Brazilian patients for the detection of inborn errors of metabolism. Eur J Pediatr., 1997; (156):650-4.

19. Shapira E, Blitzer MG, Miller JB, Africk DK. Biochemical Genetics. A laboratory manual. New York, Oxford University Press, 1989.

20. Smith P. K., Krohn, R. I., Hermanson, G. T., Mallia, A. K., Gartner, F. H., Frovenzano, M. D., Fujimoto, E. K., Goeke, N. M., Olson, B. J. y. Klenk, D. C Measurement of Protein Using Bicinchoninic Acid. Annal Biochem., 1985; (150): 76-85.

21. Besley G, Gatt S. Spectrophotometric and fluorimetric assays of galactocerebrosidase activity, their use in the diagnosis of Krabbe's disease. Clin Chimi Acta., 1981; (110):19-26.

22. Giles L., Cooper A., Fowler B., Sardharwalla I. B., Donnai $P$ Krabbe Disease: First Trimester Diagnoss confirmed on cultured amniotic fluid cells and fetal tissues. Prenat Diagn., 1987; (7):329-32.

23. Wiederschain, G., Raghavan, S. y Kolodny, E Characterization of 6-hexadecanoylamino-4-methylumbelliferyl-D-galactopyranoside as fluorogenic substrate of galactocerebrosidase for the diagnosis of Krabbe disease. Clin Chim Acta., 1992; (205):87-96.

24. Shapira C, Beaudet A, Sly W, Valle D. The metabolic and molecular bases of inherited disease. 7th edition McGraw Hill 1995; p.2465-94.

25. Uribe A. Estudio Bioquímico de los desórdenes del metabolismo de los mucopolisacáridos en Colombia. [diseertation] Bogotá - Colombia: Universidad de los Andes., 2002; p. 125-127.

26. Uribe, A., Ayala, A., España, M., Arevalo, I., Pacheco, N., \& Garcia, L. M. J. $\beta$-Galactosidase Deficiency in Colombia: Report of 20 Patients Detected Using Dried Blood Spot Samples. Journal of Inborn Errors of Metabolism and Screening., 2015;(3):3- 4

27. Moses LE, Shapiro D, Littenberg B. Combining independent studies of a diagnostic test into a summary ROC curve: Data-analytic approaches and some additional considerations. Stat Med., 1993; (12):1293 - 1316.

28. Burgueño MJ, Garcia-Bastos JL, Gonzalez-Buitrago JM. Las curvas ROC en la evaluación de las pruebas diagnósticas. Med Clin (Brac)., 1995; (104):661-70.

29. Uribe A., Espinosa E., Echeverry O., Solano M., España M., Ayala A. Tamizaje para enfermedades neurodegenerativas. Resultados de cuatro años de Investigación. Acta Neurol Colomb., 2002, (18):3-17.

30. Svennerholm L, Vanier MT, Häkansson G, Mänsson JE. Use of Leukocytes in Diagnosis of Krabbe disease and detection of carriers. Clin Chim Acta., 1981; (112):33342.

31. Argumedo, N., Uribe, A. Leukocytary Hexosaminidase Valoration: A Study in Control Population and Individuals with Neurodegenerative Illnesses. Molecular Genetics and Metabolism., 2012; 105(2), S18.

32. Krivit W, Shapiro EG, Peters C, Wagner JE, Cornu G, Kurtzberg J, Wenger DA, Kolodny EH. Hematopoietic stem-cell transplantation in globoid-cell leukodystrophy. The New England Journal of Medicine., 1998; (338): 1119 - 26.

33. Neri M, Ricca A, Di Girolamo I, Alcala B, Cavazzini FC, Orlacchio A, Martino S. Naldini L, Gritti A. Neural Stem Cell Gene Therapy Ameliorates Pathology and Function. Stem Cells., 2011; (29):1559 - 71.

34. Kondo, Y., Wenger, David A., Gallo, V., y. Duncan, Ian D Galactocerebrosidase deficient oligodendrocytes maintain stable central myelin by exogenous replacement of the missing enzyme in mice. PNAS., 2005; (102):18670 -5. 
35. Biswas, Sangita; Le Vine, Steven M. Substrate-reduction therapy enhances the benefits of bone marrow transplantation in young mice with globoid cell leukodystrophy. Pediatric research., 2002; 51(1):40-47.

36. Hawkins-Salsbury, J. A., Shea, L., Jiang, X., Hunter, D.
A., Guzman, A. M., Reddy, A. S., ... \& Sands, M. S. Mechanism-based combination treatment dramatically increases therapeutic efficacy in murine globoid cell leukodystrophy. Journal of Neuroscience., 2015; 35 (16), 6495-6505. 